\title{
Detection of Electrode Proximity to the Cochlea Wall Based on Impedance Variation: a Preliminary Computational Study
}

\author{
Enver Salkim, Majid Zamani, Dai Jiang, and Andreas Demosthenous \\ Electronic and Electrical Department, University College London, Torrington Place, WC1E 7JE, London, UK.
}

\{E-mail: e.salkim; a.demosthenous@ucl.ac.uk\}

\begin{abstract}
The electro anatomy of the cochlea plays a crucial role in hearing, where damage to the cochlea may cause hearing loss. Cochlear implants provide hearing to severe or profound hearing-impaired individuals. The accurate insertion of electrodes into the cochlea is important. If misplaced it may lead to further damage (insertion trauma). Visual inspection of the electrode array insertion is limited and relies on the experience of the surgeon. Assisted real time guidance in positioning the electrode array in the cochlea during insertion is needed. Using an advanced computational model of the cochlea accounting for different tissue layers, impedance variations at different electrode distances from the cochlear wall were simulated. Preliminary simulations suggest that the variations may be used to detect the proximity of the electrodes to the cochlea wall.
\end{abstract}

Keywords-Cochlear implant, computational models, electrode proximity, impedance measurement.

\section{INTRODUCTION}

The cochlea has a vital role in generating a sense of hearing. Sound vibrations are converted to nerve impulses by hair cells in the cochlea. These are transmitted to the brain through the auditory nerve to be processed. Damage to the hair cells in the cochlea leads to sensorineural hearing loss. A cochlear implant (CI) is a modern neural prosthesis designed to restore hearing loss by electrical stimulation of the auditory nerve fiber. Using an electrode array inserted in the scala tympani of the cochlea, the implant delivers modulated electric stimuli directly to the residual auditory nerve fibers, thus replacing the function of the damaged hair cells [1], [2].

The quality of restored hearing sensation is strongly related to the quality of surgery of CI implantation, particularly the optimum positioning of the electrode array inside the delicate cochlea without damage. It has been shown that the placement of the electrodes close to the auditory nerve fibers is of crucial importance for effective electrical stimulation However, if during the implantation procedure the electrode array touches the hair cells that are nearby to scala tympani [3], [4], it may lead to damage and hearing loss. It has been shown that the location of the electrode array relative to the cochlear wall has a strong effect on the distribution of electrical signals and the excitation pattern of the auditory nerve [5], [6].

Currently, the visual inspection by the surgeon of the cochlear electrode array position is limited. Although

This work was supported by UCL Innovation and Enterprise with EPSRC IAA 2017-2020 funding, and Advanced Bionics Ltd. post-operative computed tomography (CT) is an option, it is not desirable due to the radiation risk [7]. Positioning based on impedance measurement may be an option to monitor the proximity of the electrode array to the inner cochlear wall in real-time to prevent damage. For this it is necessary to relate impedance measurements to the distance of the electrodes from the cochlea wall which is not feasible by experiment.

In this paper the proximity of the inner cochlea wall to the electrodes, and the effect of tissue parameters [8], [9] by way of impedance measurements, is investigated using computational modes. They are implemented using finite element methods (FEM). It involves volume conductor models which represents different anatomical structures and electrodes by their conductivities and appropriate boundary conditions. Micro CT $(\mu \mathrm{CT})$ has been used to measure with high precision the cochlea internal dimensions and assess the variability of different cochleae [10], [11]. For this study, a three-dimensional (3D) volume conductor model of the human cochlea was generated based on average statistical distributions of its layers obtained from datasets. A 3D model of the electrode array was generated based on Advanced Bionic electrodes (Hannover, Germany). The impact of the proximity variation of the electrodes in the scala tympani was evaluated to determine whether the position of the electrode array could be predicted from impedance measurements.

\section{MATERIALS AND METHODS}

\section{A. Human Cochlea Modelling}

The human cochlea volume conductor was developed based on the statistical distribution of the sample set of $\mu \mathrm{CT}$ data as shown in Fig. 1(a). As the electrode array is inserted into the scala tympani, it is important to include in the volume conductor the tissue layers which are in its vicinity. Scala tympani and vestibuli, basal membrane, spiral ligament and spiral ganglion were developed based on their approximate statistical variations [11], [12]. The centre line of each layer was parameterised based on Archimedean spiral geometry using analytic equations and their derivatives. The spiral equations were converted from a polar to a Cartesian coordinate system to express each equation in a parametric form. This transformation allows modification of a set of desired parameters in the Cartesian coordinate system. The initial radius of the spiral $\left(\alpha_{\mathrm{i}}\right)$, its final radius $\left(\alpha_{\mathrm{f}}\right)$, and the desired number of turns (2.5 turns $=5 \pi$ for an average cochlea model) were 


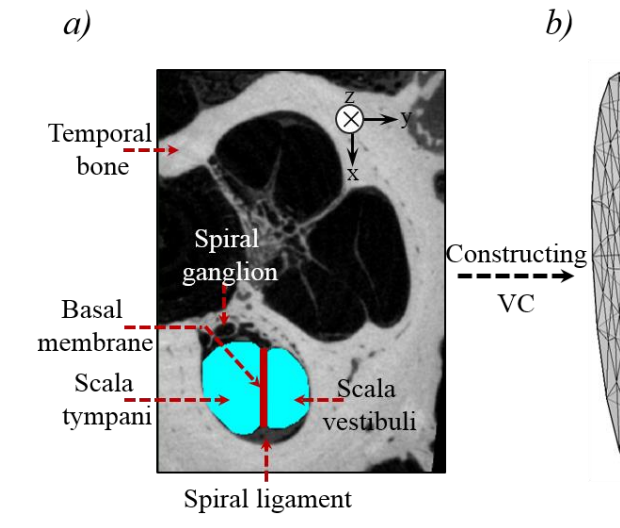

b)
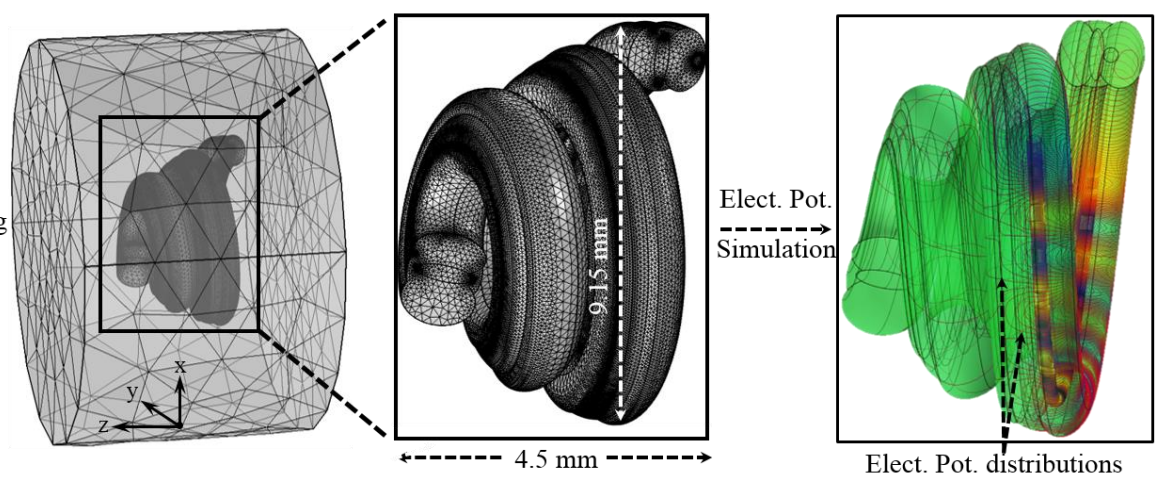

Fig. 1. Cochlea and structures in vicinity modelling and electrical potential simulation. (a) The layers were modelled based on their average statistical values in COMSOL. (b) These were then discretized and electrical potentials were solved using FEM. Since the thickness of the Stria vascularis is relatively small, it was incorporated in the models as a boundary condition. The width of the first turn of the cochlea $(9.15 \mathrm{~mm})$ and total height $(4.5 \mathrm{~mm})$ are shown. The distributions of the electrical potentials within the volume conductor (VC) are shown.

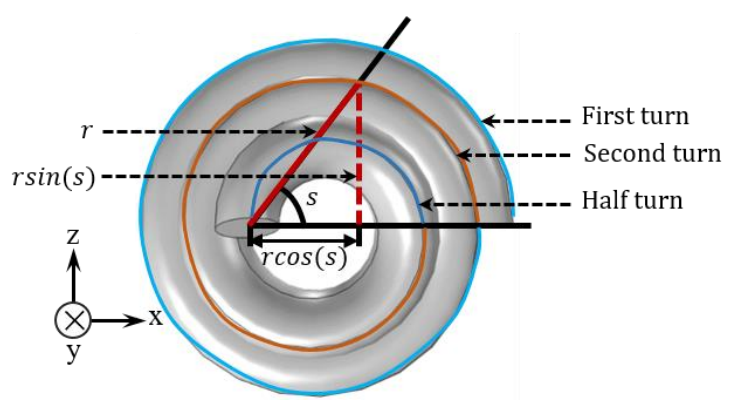

Fig. 2. 3D model of the scala tympani. The center line of the cochlea model was generated from an Archimedean spiral equation that can be described in both polar $(r, r \sin (s)$, $r \cos (s))$ and Cartesian coordinates. The associated equations are run up to $s=5 \pi$, the average rotation in the human cochlea.

defined in COMSOL Multiphysics v5.5a (COMSOL, Ltd., Cambridge, U.K.). The spiral start and end angles were set based on the average statistical variation of the cochlea [11], [12]. In COMSOL, the work plane was defined by a parametric curve with associated Archimedean equations, as shown in Fig. 2. Varying angles were entered into the parametric curve expression field to generate the Cartesian coordinates $(x, y, z)$ using Eqs. (1) to (4).

$$
\begin{gathered}
x=(\alpha+\beta * s) * \cos (s) \\
y=(\alpha+\beta * s) * \sin (s) \\
z=h_{\max } * s / \theta_{f} \\
\beta=\left(\alpha_{\mathrm{f}}-\alpha_{\mathrm{i}}\right) / 2 \pi \mathrm{n}
\end{gathered}
$$

where, $\alpha$ is spiral radius, $\beta$ the growth rate, $s$ the varying parameter, $h_{\max }$ the length of the cochlea, $\theta_{f}$ the spiral final angle and $n$ the turn numbers.

The 3D models of the scala tympani and vestibuli were generated based on elliptic geometry with associated radii using a parametric sweep function by following a spiral curve in COMSOL Multiphysics. These functions were applied up to the average number of turns ( 2.5 turns $=5 \pi)$ of the cochlea as shown in Fig. 2. The remaining layers were constructed using similar functions and using Boolean operations to remove any intersections. Since it is not possible to observe the variation of the medium layer on the datasets due to its thinness, and the conductivity of this layer is similar to the vestibuli layer, it was modelled as a part of the vestibuli layer. The thickness of the basilar membrane was doubled based on the $\mu \mathrm{CT}$ data. The total length and total width $(\mathrm{L} \times \mathrm{W}=4.5 \times 9.15 \mathrm{~mm})$ of the cochlea were set to be slightly longer and wider to prevent self-intersection as indicated in Fig. 1(b). Bony structures were modelled in a smooth geometry to reduce the computation size as shown in Fig. 1(b). Since the fibrous tissue and bone layer have similar conductivity, the fibrous tissue was designed as a part of the bony structure. The stria vascularis layer is comparatively thin and was modelled as 'contact impedance' during all simulations.

\section{B. Electrode Array Modelling}

Electrode array modelling was designed based on the Advanced Bionic Slim 1j electrode array with 16 platinum electrodes to conduct stimulation currents to different parts of the cochlea as shown in Fig. 3(b). The electrode size $(\mathrm{L}=0.4 \mathrm{~mm} ; \mathrm{W}=0.5 \mathrm{~mm})$, the spacing of electrodes $(\sim 1.1 \mathrm{~mm})$ and the total length of 16 electrodes $(\sim 17 \mathrm{~mm})$ were chosen from literature. Although the electrode tip and base diameter are different, the electrodes were designed using a base diameter $(0.4 \mathrm{~mm})$ in this study. The 16 platinum electrodes are designed to face the inner 
a)
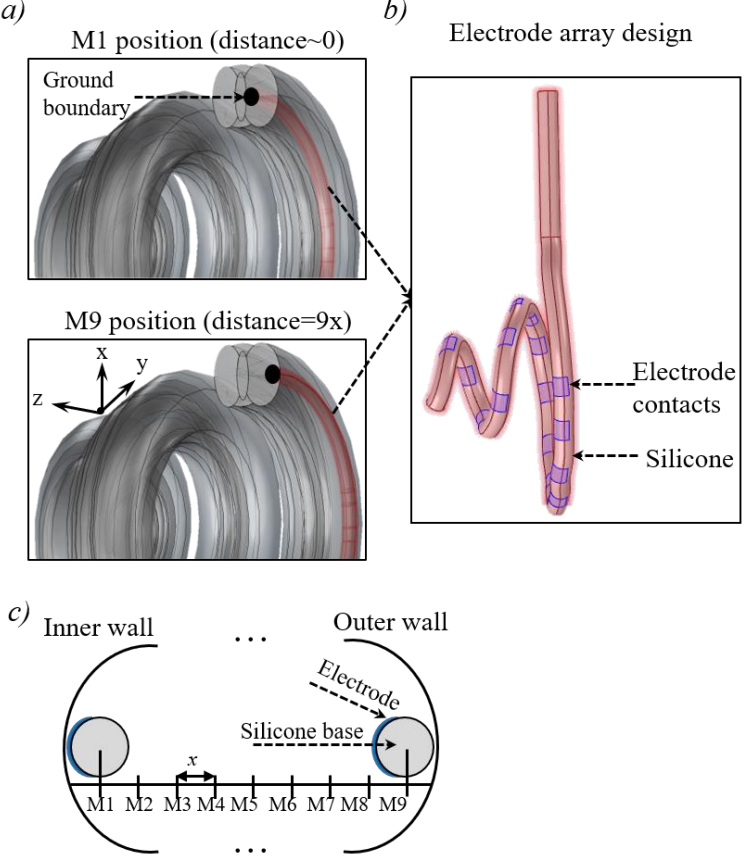

Fig. 3. (a) electrode array in the human average cochlea model. $x$ shows relative distance of the electrode array to the cochlear wall. The ground boundary is highlighted. Sample 1 shows the electrode array touching the cochlea wall and sample 2 represents the furthest model. (b) Electrode array design, the electrode contacts (16 discrete contacts) are highlighted. (c). Diagram showing the M1 to M9 positions measured in the cochlea for electrode 8 .

cochlear wall with soft silicone that supports the electrodes [Fig. 3(b)]. The electrode array was modelled inside the cochlea by interpolating the centre points of the scala tympani and using the sweep function in COMSOL. The centre point model was generated by calculating a variable cross-section of the scala tympani along with the spiral shape of the cochlea and stored as the $x$, $y$ and $z$ coordinates of the geometric centre of each crosssection. These were interpolated with a relatively high number in MATLAB v.R2019b (MathWorks, Inc., Natic M, USA) to obtain a smooth curve. The data was imported to COMSOL to generate the 3D model of the electrode array using the sweep function. Since the electrodes are relatively thin, they were designed as a boundary surface. To parameterise the distance between the electrode array and cochlea wall, the electrode array was shifted in the $z$ direction in incremental steps until the silicone base of the electrode array touched the outer wall of the scala tympani as shown in Fig. 3(c). This resulted in nine different scenarios. The electrical potential due to a current input at the selected electrode were simulated and the impedance was measured for each $\mathrm{M}$ position. Note that electrode 8 was used for all measurements.
TABLE I. TISSUE CONDUCTIVITIES

\begin{tabular}{lcr}
\hline \hline $\begin{array}{l}\text { Tissue } \\
\text { layer }\end{array}$ & $\begin{array}{c}\text { Conductivity } \\
(\mathrm{S} / \mathrm{m})\end{array}$ & Source \\
\hline $\begin{array}{l}\text { Scalas } \\
\text { (Perilymph) }\end{array}$ & 1.43 & {$[14]$} \\
\hline $\begin{array}{l}\text { Basilar } \\
\text { membrane }\end{array}$ & 0.0125 & {$[14]$} \\
\hline $\begin{array}{l}\text { Spiral } \\
\text { ligament }\end{array}$ & 1.67 & {$[14]$} \\
\hline $\begin{array}{l}\text { Stria } \\
\text { vascularis }\end{array}$ & 0.005 & {$[14]$} \\
\hline $\begin{array}{l}\text { Spiral } \\
\text { ganglion }\end{array}$ & 0.33 & {$[13]$} \\
\hline Bone & 0.0156 & {$[14],[13]$} \\
\hline Silicone & $1 \mathrm{e}-7$ & {$[13]$} \\
\hline Platinum & 1000 & {$[13]$} \\
\hline
\end{tabular}

\section{Volume Conduction Simulation}

FEM was used to calculate the electrical potentials within the volume conductor by discretising the domains using free tetrahedral elements. The regions of interest (mainly electrode array, basilar membrane and scala tympani) were more finely meshed, while the rest of the region was relatively coarsely meshed to obtain accurate potential distributions in a reasonable time. This resulted in 4 to 6 million tetrahedral elements and about 9 million degrees of freedom. In all models, a current was applied to electrode 8 . The current passes from the electrode through the surrounding medium and returns to the ground. The electrode-tissue interface contact impedance was assumed to be zero and appropriate continuity conditions were implemented at the boundary of the different domains to provide a unique solution [8].

The conductivity domains in the volume conductor are listed in Table I which were based on low frequency measurements. The conductivities of the tissue layers were assumed to be isotropic.

\section{RESULTS}

The impedance variation for the proximity of nine different electrode settings to the cochlear wall based on the average cochlear model are shown in Fig. 4. It is clear that there is a relationship between electrode proximity to the cochlear wall and recorded impedance amplitudes. In general, the results showed that the impedance variation is increased as the electrode nears the inner or outer cochlear wall. When the electrode base approaches and nearly touches the outer wall, the impedance also increases but to a lesser degree. There is a distinguishable impedance difference between M1, M2 and M3. The impedance variation for M3 to M8 (which are far from both sides the scala tympani walls) shows relatively lower variations.

\section{DISCUSSION}

Advanced computational modelling facilitates a depth and scale of investigation that may not possible in experimental tests. Numerical methods have been used as a tool to study electrical impedances within the volume 


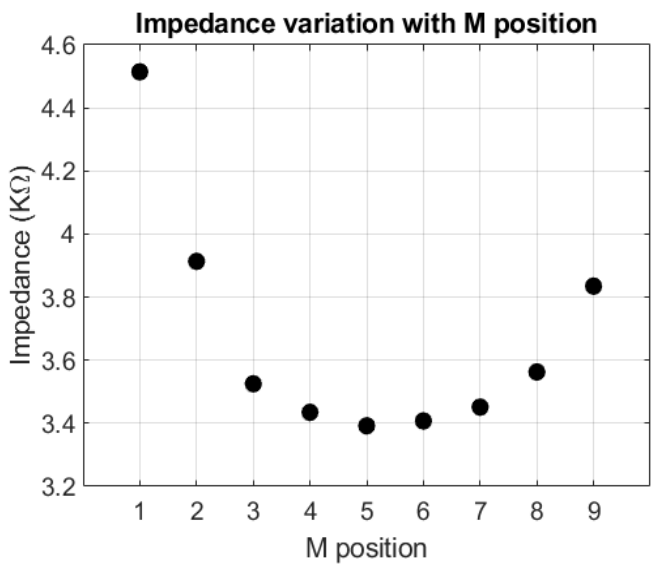

Fig. 4. Impedance variation at different electrode proximity positions [see Fig. 3 (c)].

conductors. The neuromodulator can be designed and developed using these sophisticated computational methods [5], [8], [13].

In this study the volume conductor of the cochlea, the tissue layers in its vicinity and the implanted stimulation electrodes were constructed to analyse electrode impedance variations at different electrode proximities to the cochlear wall. The results show the variation of impedance at different electrode positions in the scala tympani which is in agreement with the previous studies [7]. However, those results were based on a plastic cochlea model and in vitro saline measurements.

A more detailed cochlea model based on $\mu \mathrm{CT}$ representing a large group of individuals and models of the surrounding tissues, results in an accurate impedance variation matrix used to determine whether electrode proximity to the cochlea wall can be detected, based on impedance variations in real time. The results in Fig. 4 which include surrounding tissues, show a useful difference in impedance variation with proximity. Compared to the centre position M5, M2 showed about $20 \%$ and M1 about $40 \%$ increase in impedance. This suggests that the variations in impedance can be used as a guide during electrode array insertion.

\section{CONCLUSION AND Future WORK}

A 3D model of the cochlea has been generated to identify the position of the electrode array based on the impedance variation at different electrode proximities. The results show that when an electrode is moved away from the central positions the impedance measurements are increased. When an electrode is close to the inner cochlear wall, there is a 20 to $40 \%$ impedance increase which suggests that the impedance measurement could

guide the clinician to position the electrode in the cochlea avoiding touching sensitive structures (e.g., hair cells).

Although the results are promising, impedance variations on only one electrode have been tested. The other 15 electrodes need evaluating. The anatomical layers in the cochlea and in its vicinity, which are used in the volume conductor model of the detailed human cochlea, require further information from many different individuals to obtain more accurate results which will provide useful real time impedance measurements.

\section{REFERENCES}

A. Dhanasingh and C. Jolly, "An overview of cochlear implant electrode array designs," Hear. Res., vol. 356, pp. 93 103, 2017.

K. Dang, "Electrical conduction models for cochlear implant stimulation," 2017.

L. K. Holden et al., "Factors affecting open-set word recognition in adults with cochlear implants," vol. 34, no. 3, pp. 342-360, 2014.

K. S. Min, S. B. Jun, Y. S. Lim, S. I. Park, and S. J. Kim, "Modiolus-hugging intracochlear electrode array with shape memory alloy," Comput. Math. Methods Med., vol. 2013, 2013.

T. H. and J. J. H. T. K. Malherbe, "Constructing a threedimensional electrical model of a living cochlear implant user's cochlea," Training, vol. 4179, no. December 2015, p. 53, 2016.

J. H. M. Frijns, J. J. Briaire, and J. J. Grote, "The importance of human cochlear anatomy for the results of modiolushugging multichannel cochlear implants," Otol. Neurotol., vol. 22, no. 3, pp. 340-349, 2001.

C. K. Giardina, E. S. Krause, K. Koka, and D. C. Fitzpatrick, "Impedance measures during in vitro cochlear implantation predict array positioning," IEEE Trans. Biomed. Eng., vol. 65, no. 2, pp. 327-335, 2018.

E. Salkim, A. Shiraz, and A. Demosthenous, "Impact of neuroanatomical variations and electrode orientation on stimulus current in a device for migraine: a computational study," J. Neural Eng., vol. 17, no. 1, p. 016006, 2019.

E. Salkim, A. Shiraz, and A. Demosthenous, "Influence of cellular structures of skin on fiber activation thresholds and computation cost In fl uence of cellular structures of skin on fi ber activation thresholds and computation cost," Biomed. Phys. Eng. Express, vol. 5, no. 1, p. 015015, 2018.

N. Gerber et al., "A multiscale imaging and modelling dataset of the human inner ear," Sci. data, vol. 4, p. 170132, 2017.

E. Avci, T. Nauwelaers, T. Lenarz, V. Hamacher, and A. Kral, "Variations in microanatomy of the human cochlea," J. Comp. Neurol., vol. 522, no. 14, pp. 3245-3261, 2014.

E. Erixon, H. Högstorp, K. Wadin, and H. Rask-Andersen, "Variational anatomy of the human cochlea: Implications for cochlear implantation," Otol. Neurotol., vol. 30, no. 1, pp. 14 22, 2009. implants: A review of their development and how they could support management and maintenance of cochlear implant performance," Network: Computation in Neural Systems, vol. 27, no. 2-3. pp. 67-106, 2016.

C. C. Finley and M. W. White, "Models of Neural Responsiveness to Electrical Stimulation," Springer- Verlag, 1990, pp. 55-99. 